\title{
La Escala de Ansiedad y Depresión Hospitalaria (HAD) en fibromialgia: Análisis de sensibilidad y especificidad
}

\section{Hospital Anxiety and Depression Scale (HADS) in fibromyalgia: Sensitivity and specificity analysis}

\author{
Víctor Cabrera \\ Maite Martín-Aragón \\ María del Carmen Terol \\ Rosa Núñez \\ María de los Ángeles Pastor \\ Universidad Miguel Hernández de Elche, España
}

(Rec: 31 marzo 2014 / Acept: 24 julio 2015)

\begin{abstract}
Resumen
La Sociedad Española de Reumatología (SER) (Rivera et al., 2006) recomienda la Escala de Ansiedad y Depresión Hospitalaria (Hospital Anxiety and Depression Scale - HADS, Zigmond y Snaith, 1983) para la evaluación clínica de ansiedad y depresión en Fibromialgia (FM). Sin embargo, no constan índices de sensibilidad/especificidad ni puntos de corte en FM. Con el objetivo de comprobar aplicabilidad, propiedades psicométricas y sensibilidad/especificidad del HAD en FM, se evaluó a 131 pacientes ambulatorios del Hospital de San Vicente (Alicante, España), utilizando como criterios externos BDI y STAI-E. Se analizaron ítems, consistencia interna y estructura factorial mediante análisis confirmatorio y puntos de corte con curvas ROC. Se replicó la estructura de dos factores. La fiabilidad es adecuada (HADA=.80, HADD=.85). Se muestran diferentes puntos de corte con sensibilidad/especificidad superior al 70\% para cada subescala. El HAD presenta propiedades psicométricas óptimas en población FM, mostrándose útil como instrumento de "screening" de sintomatología ansioso-depresiva en esta población.

Palabras clave: HAD, fibromialgia, análisis psicométrico, sensibilidad y especificidad.
\end{abstract}

\begin{abstract}
Spanish Society of Rheumatology (SER) (Rivera et al., 2006) suggests the Hospital Anxiety and Depression Scale (HADS, Zigmond y Snaith, 1983) for clinical assessment of anxiety and depression in fibromyalgia (FM). However, there is no HAD-FM cut-off yet. Aiming to assess applicability, psychometric properties and sensitivity/specificity of HADS in FM, 131 outpatients were assessed at San Vicente Hospital (Fibromyalgia Service, Alicante, Spain), using like external criteria BDI and STAI-E. Item analysis was performed, calculating the internal consistency and factor structure study by confirmatory factor analysis (CFA). The cut-off points were found by ROC curves. Two-factor structure was replicated. Reliability is adequate $(\mathrm{HADA}=.80, \mathrm{HADD}=.85)$. Several cut-off points are showed with sensitivity and specificity levels $>70 \%$ for both subscales. HADS has optimal psychometric properties in FM and its useful as a screening tool to assess anxious-depressive symptoms in FM patients.
\end{abstract}

Keywords: HADS, fibromyalgia, psychometric analysis, sensitivity, specificity.

\footnotetext{
Correspondencia: Víctor Cabrera Perona. E-mail: vcabrera@umh.es, Dpto. de Psicología de la Salud. Campus de San Juan, Universidad Miguel Hernández de Elche. 03550 San Juan (Alicante, España). Tfno: 96.542.97.25.

Agradecimientos: Este estudio forma parte del Programa de Formación de Profesorado Universitario FPU. Convocatoria 2010. Ministerio de Educación. Referencia: AP2010-1870.
} 


\section{Introducción}

En población española se estima una prevalencia de Fibromialgia (FM) del 2.4\% (Estudio EPISER-Valverde, 2007), con una media de padecimiento de dolor hasta diagnóstico de entre 4-6 años (Peñacoba, 2009), lo que sitúa a la FM como un problema de salud pública debido al número de visitas médicas, consumo de fármacos y gasto sanitario (Sicras, Blanca, Navarro y Rejas, 2009a). La FM es una enfermedad crónica de etiología desconocida, caracterizada por dolor músculo-esquelético generalizado acompañado de sintomatología diversa como fatiga, alteraciones de sueño, rigidez, parestesias (entre otras) y trastornos de ansiedad y depresión (Miró, 2003; Wolfe et al., 1997). Estos trastornos son frecuentemente referidos en estas pacientes (Moix, 2005), presentándose en mayor proporción que la esperada (Comeche, Martín, Rodríguez-Muñoz, Ortega, Díaz y Vallejo, 2010; Ruiz, Muñoz, Pérula de Torres y Aguayo, 1997) y relacionándose con el mantenimiento y exacerbación del dolor (Monsalve, Cerdá-Olmedo, Mínguez y De Andrés, 2000).

La prevalencia de ansiedad y depresión en FM oscila entre el 30\%-68\% en distintos momentos de la evolución de la enfermedad (Bennett, 2002; Epstein et al., 1999; González, Elorza y Failde, 2010; Revuelta, Segura y Paulino, 2010). Aunque estas afectaciones emocionales no parecen ser la causa de la FM, agravan los síntomas principales y empeoran su curso y evolución (Revuelta, Segura y Paulino, 2010), pudiendo afectar a la adhesión terapéutica y dificultar el diagnóstico (Katon, 1996; Pérez-Pareja, Borrás, Palmer, Sesé, Molina y Gonzalvo, 2004), por lo que son los propios profesionales quienes desde su práctica clínica con estas pacientes demandan instrumentos de "screening" que sirvan de apoyo para la evaluación diagnóstica. La Sociedad Española de Reumatología (SER), en su documento de consenso sobre la fibromialgia, recomienda la escala de Ansiedad y Depresión Hospitalaria (HAD, Hospital Anxiety and Depression Scale-HADS, Zigmond y Snaith, 1983), para evaluar dicha sintomatología en estas pacientes, frente a instrumentos alternativos como el BDI (Beck Depression Inventory, Beck, 1978; Beck, Steer y Harbin, 1988), CES-D (Center for Epidemiologic Studies-Depression Scale, CES-D, 1977; Soler, Pérez, Puigdemont, Pérez, Figueres y Álvarez, 1997) o STAI (State Trait Anxiety InventorySTAI, Spieldberger, Gorsuch y Lushene, 1982) (Rivera et al., 2006). El HAD es una de las pruebas más utilizadas para detectar malestar emocional en pacientes hospitalarios no psiquiátricos, con enfermedad física (Brennan et al., 2010;
Tyrer y Methuen, 2007). Es un instrumento corto (14 ítems) que ha mostrado su fiabilidad y validez y ha sido utilizado tanto para el diagnóstico como para medir la gravedad del trastorno (Rivera et al., 2006). Algunas de las ventajas del HAD son su brevedad y la exclusión de ítems de carácter somático que "depura" la evaluación en pacientes con sintomatología física (pérdida de apetito o energía, fatiga, insomnio, entre otros). Esto hace que el HAD se considere especialmente útil para adaptarse en diferentes contextos de salud y hospitalarios (Terol et al., 2007).

Distintos trabajos sobre el HAD en enfermos físicos analizan su estructura factorial y su sensibilidad y especificidad (Bjelland et al., 2002; Brennan et al., 2010; Cosco, Doyle, Ward y McGee, 2011; Morse, Kendall y Barton, 2005). También muestran su adecuación y aplicabilidad en diferentes tipos de población y contexto de enfermedad, confirmando mayoritariamente sus factores originales (Mykletun, Stordal y Dahl, 2001; Spinhoven et al., 1997; entre otros). En población española, los estudios de comprobación de las propiedades psicométricas del instrumento han utilizado sujetos sanos (Terol et al., 2007), enfermos físicos (Costa, Pérez, Salamero y Gil, 2009; Noguera et al., 2009; Monsalve, Soriano e Ibáñez, 2001; López-Roig et al., 2000), concretamente dos de ellos con pacientes de FM (Luciano, Barrada, Aguado, Osma y García-Campayo, 2013; Vallejo, Rivera, EsteveVives y Rodríguez-Muñoz, 2012), enfermos mentales (Chivite, Martínez, Pérez y Peralta, 2007; De las Cuevas, García-Estrada y González de Rivera, 1995; Tejero et al., 1986) y también poblaciones mixtas (Herrero et al., 2003; Ibáñez y Caro, 1992; Quintana et al., 2003). Los resultados obtenidos con muestras españolas de enfermos físicos han mostrado las buenas propiedades psicométricas del HAD y también han replicado la estructura bifactorial propuesta por Zigmond y Snaith (1983) (Costa et al., 2009; Herrero et al., 2003; López-Roig et al., 2000; Luciano et al., 2013). Sin embargo, estos trabajos sólo muestran puntos de corte para población oncológica (Costa et al., 2009; López-Roig et al., 2000) y para una muestra hospitalaria (neurocirugía, enfermedad pulmonar, cardiología, neurología y enfermedades infecciosas) (Herrero et al., 2003). Éstos oscilan entre 3+ (López-Roig et al., 2000) y $5+$ (Herrero et al., 2003; Terol et al., 2007) para depresión, con sensibilidad superior al $70 \%$ y especificidad variable $(60 \%-85 \%)$ y de $8+9+$ (Costa et al., 2009; Herrero et al., 2003; López-Roig et al., 2000) en ansiedad con sensibilidad y especificidad superiores al $70 \%$.

A la vista de la revisión realizada en población española (Terol, Cabrera y Martín-Aragón, 2015), la recomendación de la SER (Rivera et al., 2006) sobre el HAD como instrumento de 
elección en FM, y la alta prevalencia de ansiedad y depresión en estas pacientes, el objetivo de este trabajo es comprobar la aplicabilidad y propiedades psicométricas del HAD analizando su sensibilidad y especificidad en pacientes con FM.

\section{Método}

\section{Participantes}

Evaluamos 131 mujeres diagnosticadas de FM recibiendo tratamiento ambulatorio en la unidad de fibromialgia del Hospital de San Vicente. Los criterios de inclusión fueron: presentar diagnóstico confirmado de FM según criterio del American College of Rheumatology (ACR) (Wolfe et al., 1990), comprender los cuestionarios y el compromiso de participación en la investigación.

La media de edad fue de 50.15 años (DT=11.1), mayoritariamente casadas (68.7\%). El 82.4\% de las pacientes habían sido diagnosticadas de FM antes de la llegada a la unidad, con edad media de inicio del dolor de 36.2 años

Tabla 1. Descripción sociodemográfica de la muestra.

\begin{tabular}{lcc}
\hline N=131, Edad media=50.1 (DT=11.1) & N & $\%$ \\
\hline Estado civil & & \\
\hline Casada & 90 & 68.7 \\
Soltera & 20 & 15.3 \\
Separada-divorciada & 17 & 13 \\
Viuda & 4 & 3.1 \\
\hline Nivel educativo & & \\
\hline No lee/no escribe & 5 & 3.8 \\
Lee/escribe & 7 & 5.3 \\
Estudios primarios & 55 & 42 \\
Estudios medios & 48 & 36.6 \\
Estudios superiores & 14 & 10.7 \\
\hline Situación laboral & & \\
\hline Activo & 52 & 39.7 \\
Ama de casa & 43 & 32.8 \\
En desempleo & 24 & 18.3 \\
Incapacidad laboral temporal & 1 & 0.8 \\
Incapacidad laboral permanente /Jubilación & 8 & 6.1 \\
Baja laboral & 18 & 13.7 \\
Pendiente de juicio laboral & 10 & 7.6 \\
\hline & & \\
\hline
\end{tabular}

(DT=11.7). El resto de características sociodemográficas se muestran en la Tabla 1.

\section{Variables e instrumentos}

Ansiedad y depresión: Escala de ansiedad y depresión hospitalaria (HAD) (Hospital Anxiety and Depression Scale: Zigmond y Snaith, 1983; adaptación de Terol et al., 2007). Cuestionario autoaplicado de 14 ítems. Dos subescalas de 7 ítems cada una, en escala Likert 0-3. HADA ítems impares, HADD ítems pares, con rango de puntuación en cada subescala de 0-21. Mayor puntuación, mayor ansiedad y depresión. Para ambas subescalas, los autores sugieren que las puntuaciones superiores a once indicarían "caso" y mayores de ocho se considerarían "caso probable" (Zigmond y Snaith, 1983). La consistencia interna para población española en un estudio con fibromialgia fue HADA $\alpha=.83$ y HADD $\alpha=.87$ (Vallejo, Rivera, Esteve-Vives y RodríguezMuñoz, 2012).

Ansiedad: Inventario de ansiedad estado-rasgo (State Trait Anxiety Inventory-STAI: Spieldberger, Gorsuch y Lushene, 1982). Dos subescalas de 20 ítems cada una, con respuesta 0 a 3 . Presenta una consistencia interna entre $\alpha=.90$ y $\alpha=.93$ para ansiedad-estado y entre $\alpha=.84$ y $\alpha=.87$ para ansiedad-rasgo (Spieldberger, Gorsuch y Lushene, 1982). Para este estudio, el valor de la subescala de ansiedad-estado fue $\alpha=93$. De esta subescala, se tomó el percentil 75 (puntuación 31 en mujeres) como criterio clínicamente significativo de ansiedad elevada (Echeburúa, 2004). Además, en función de la media y desviación típica (DT) obtenida en nuestra muestra $(32.76 \pm 11.27)$ se calculó el punto de corte para el intervalo de $+1 \mathrm{DT}$, coincidiendo con el percentil 90 (puntuación 44 en mujeres) y valores extremos de ansiedad.

Depresión: Inventario de depresión de Beck (Beck Depression Inventory-BDI: Beck, 1978; Beck, Steer y Harbin, 1988; adaptación española de Vázquez y Sanz, 1997). Escala de 21 ítems con respuesta 0 a 3 puntos y rango de puntuación de 0-63. Su consistencia interna para poblaciones psiquiátricas y no psiquiátricas, $\alpha=.76$ y $\alpha=.95$ respectivamente (Beck, 1978; Beck, Steer y Harbin, 1988). Para este estudio, el valor fue $\alpha=.88$. Los puntos de corte para el diagnóstico de depresión son: 0-9: ausencia de depresión, 10-18: depresión leve, 19-29: depresión moderada-severa y 30-63: depresión grave. Otro punto de corte recomendado, en particular en el caso de sujetos con dolor crónico, es $=21$ (Geisser, Roth y Robinson, 1997; Hamilton y Shapiro, 1990). 
Tabla 2. Instrumentos de evaluación.

\begin{tabular}{|c|c|c|c|c|}
\hline Variable & Instrumento & Autor & $\begin{array}{l}\text { Número } \\
\text { de } \\
\text { ítems }\end{array}$ & $\begin{array}{l}\text { Consistencia } \\
\text { interna } \\
(\alpha)\end{array}$ \\
\hline Sociodemográficas & ad-hoc & - & - & - \\
\hline Clínicas & ad-hoc & - & - & - \\
\hline Ansiedad y depresión & $\begin{array}{l}\text { HAD. Escala de ansiedad y depresión } \\
\text { hospitalaria (Hospital Anxiety and } \\
\text { Depression Scale) }\end{array}$ & $\begin{array}{l}\text { Zigmond y Snaith (1983); } \\
\text { adaptación española de López-Roig et al. } \\
\text { (2000) }\end{array}$ & 14 & $\begin{array}{c}\text { HADA }=.77 \\
\text { HADD }=.71 \\
\quad(\text { sanos }) \\
\text { HADA }=.85 \\
\text { HADD }=.87 \\
\text { (físicos) }\end{array}$ \\
\hline Ansiedad (criterio) & $\begin{array}{l}\text { STAI. Inventario de ansiedad, estado } \\
\text { y rasgo } \\
\text { (State Trait Anxiety Inventory-STAI) }\end{array}$ & $\begin{array}{l}\text { Spieldberger, Gorsuch y Lushene (1982); } \\
\text { Versión española de TEA (1982) }\end{array}$ & 20 & $\begin{array}{l}\text { STAI-E }=.90-.93 \\
\text { STAI-R }=.84-.87\end{array}$ \\
\hline Depresión (criterio) & $\begin{array}{l}\text { BDI. Inventario de depresión de Beck } \\
\text { (Beck Depression Inventory-BDI) }\end{array}$ & $\begin{array}{l}\text { Beck, 1978; Beck, Steer y Harbin, (1988); } \\
\text { Adaptación española de Vázquez y Sanz } \\
\text { (1997) }\end{array}$ & 21 & $.76-.95$ \\
\hline
\end{tabular}

HADA: Subescala de Ansiedad del HAD; HADD: Subescala de Depresión del HAD; STAI-E/R: Cuestionario de Ansiedad-Estado de Spielberger (State Trait Anxiety Inventary; 1982); STAI-R: Cuestionario de Ansiedad-Rasgo de Spielberger (State Trait Anxiety Inventary; 1982).

\section{Procedimiento}

Estudio descriptivo-correlacional de corte transversal en la unidad de fibromialgia del Hospital de San Vicente del Raspeig (Alicante). Tras la aprobación del proyecto por el comité de ética del hospital, durante 12 meses (mayo de 2011-mayo de 2012) se seleccionó a pacientes de nuevo ingreso. En la primera cita se informaba a los pacientes del objetivo, procedimiento y metodología del estudio firmando el consentimiento informado. Posteriormente se procedía a la administración de cuestionarios, que eran cumplimentados bajo supervisión de un psicólogo, con un tiempo que oscilaba entre 30-50 minutos.

\section{Análisis de los datos}

Se utilizaron los paquetes estadísticos IBM SPSS v.19 y AMOS v.20. Cálculo de la consistencia interna con el coeficiente alfa de Cronbach y análisis de relaciones con el índice de correlación de Pearson. Análisis factorial confirmatorio (AMOS v.20) con estimación por máxima verosimilitud. Se consideraron valores óptimos de los índices de ajuste: ji-cuadrado, la razón de j-cuadrado y los grados de libertad; GFI, índice de bondad de ajuste; AGFI, índice ajustado de bondad de ajuste; NFI, índice de ajuste normado; CFI, índice de ajuste comparado y RMSEA, error de aproximación cuadrático medio. (Byrne, 2010; García- Cueto, Gallo y Miranda, 1998) (ver Tabla 4). Puntos de corte, sensibilidad y especificidad se hallaron mediante curvas ROC, clasificando casos y no-casos según las puntuaciones de los criterios diagnósticos externos. Se valoró valor predictivo positivo (VPP), valor predictivo negativo (NPV) e índice de eficacia diagnóstica (AC) (Álvarez, 2007). Claves de interpretación: a) AUC: Área bajo la curva. Proporción de casos diagnosticados por el criterio externo al azar, que también lo serían por la subescala HAD; b) Sensibilidad: probabilidad de resultado positivo en el HAD de un paciente clasificado como "caso" en el criterio externo; c) Especificidad: probabilidad de resultado negativo en el HAD de un paciente clasificado como "no-caso" en el criterio externo; d) PPV: Probabilidad de padecer el trastorno un paciente clasificado como "caso" por el HAD; e) NPV: Probabilidad de no padecer la afectación un paciente clasificado como "no-caso" por el HAD; f) AC: proporción de casos correctamente clasificados (verdaderos positivos+verdaderos negativos) en el total de la muestra. 


\section{Resultados}

La puntuación media para la subescala de depresión (HADD) fue de 10.7 ( $D T=4.6$, rango: mín=1,máx=20). Para la subescala de ansiedad (HADA) fue 13.7 ( $D T=$ 4, Rango: $\operatorname{mín}=3$, máx=21). Se obtuvieron correlaciones significativas entre las subescalas del HAD y todos los criterios (ver tabla 3 ).

\section{Análisis factorial confirmatorio}

Se comprobó la estructura factorial del HAD que definieron Zigmond y Snaith (1983). Teniendo en cuenta la sensibilidad de la prueba al tamaño muestral de $\mathrm{x}^{2}$, se recurre $\mathrm{a} \mathrm{x}^{2} / \mathrm{gl}$, obteniendo 1.87 (rango aceptable 1-3), mostrando un error cuadrático medio de aproximación razonable (RMSEA<.08) y un índice de incremento comparativo con buen ajuste a los datos (CFI>.95) (ver tabla 4).

\section{Análisis de la consistencia interna}

La consistencia interna de las escalas fue de $\alpha=.80$ para la HADA y de $\alpha=.85$ para la HADD. De la valoración psicométrica de sus ítems, todos mostraron correlación ítem-test ( $r$.40) (Tabla 5). Para la ansiedad (HADA), el ítem con más baja correlación es el ítem $11(r=.45)$ y el de mayor correlación es el ítem 13 ( $r=.63)$. Para depresión (HADD), el ítem con menor correlación es el $8(r=.45)$ y el ítem con mayor correlación el $12(r=.78)$.

\section{Sensibilidad y especificidad para puntos de corte}

En la subescala HADD con criterio externo BDI $=10$ (depresión leve) se obtiene un área bajo la curva $\mathrm{AUC}=.853$, con puntos de corte de 7+ y $8+$ con índices de sensibilidad (S) y Especificidad (E) superiores a 78\%. Respecto al criterio $\mathrm{BDI}=19$ (depresión moderada) se obtiene un $\mathrm{AUC}=.862$ con punto de corte $10+(\mathrm{S}: 81 \%, \mathrm{E}: 73 \%, \mathrm{PPV}=80 \%, \mathrm{NPV}=74 \%)$. Para el criterio $\mathrm{BDI}=21$ (criterio dolor crónico) se obtiene un área bajo la curva $\mathrm{AUC}=.837$ con punto de corte de $11+(\mathrm{S}: 78 \%$, E: $71 \%$ ) y $12+(S: 73 \%, E: 75 \%, P P V=70 \%, N P V=77 \%)$. En cuanto al criterio $\mathrm{BDI}>30$ (depresión grave), con $\mathrm{AUC}=.808$, el punto de corte $13+$ presenta S:76\% y E:73\%.

Para la subescala HADA y criterio STAI-E (percentil 75), $\mathrm{AUC}=.858$, el punto de corte $12+$ muestra una $\mathrm{S}: 80 \%$ y E:68\% (PPV:91, NPV:45). El punto de corte para el percentil 90 es de $16+(\mathrm{AUC}=.755, \mathrm{~S}: 72 \%, \mathrm{E}: 68 \%)$

En las tablas 6 y 7 se presentan los valores de AUC, sensibilidad y especificidad, los valores predictivos positivo y negativo, y la eficacia diagnóstica para cada criterio.

Tabla 3. Estadisticos descriptivos y correlaciones.

\begin{tabular}{|c|c|c|c|c|c|c|c|c|}
\hline & $\mathrm{M}$ & DT & Min-Max & HADT & HADA & HADD & BDI & STAI-E \\
\hline HADT & 24.44 & 7.88 & $6-40$ & - & $.894 * *$ & $.920 * *$ & $.665 * *$ & $.480 * *$ \\
\hline HADA & 13.71 & 4 & $3-21$ & & - & $.647 * *$ & $.528 * *$ & $.460 * *$ \\
\hline HADD & 10.73 & 4.64 & $1-20$ & & & - & $.672 * *$ & $.414 * *$ \\
\hline BDI & 21.74 & 9.98 & $4-52$ & & & & - & $.656^{* *}$ \\
\hline STAI-E & 32.76 & 11.27 & $8-58$ & & & & & - \\
\hline
\end{tabular}

BDI: Inventario de Depresión de Beck (Beck Depression Iventory, Beck, 1978); HADA: Subescala de Ansiedad del HAD; HADD: Subescala de Depresión del HAD; HADT: Escala de Ansiedad y Depresión Total; Mín.: Valor mínimo; Máx.: Valor Máximo; STAI-E: Cuestionario de Ansiedad Estado de Spielberger (State Trait Anxiety Inventary; 1982).

Tabla 4. Índices de ajuste al modelo por el método de máxima verosimilitud.

\begin{tabular}{|c|c|c|c|c|c|c|c|}
\hline & $x^{2}$ & $x^{2} / \mathrm{gl}$ & GFI & AGFI & NFI & CFI & RMSEA \\
\hline Valores óptimos & $(\mathrm{p}>=.05)$ & $1-3$ & $>=.90$ & $>=.90$ & $>=.90$ & $>=.90$ & $<=.08$ \\
\hline Valores obtenidos & $142.049(\mathrm{p}=.000)$ & 1.869 & .867 & .816 & .826 & .909 & .079 \\
\hline
\end{tabular}

GFI: índice de bondad de ajuste; AGFI: índice ajustado de bondad de ajuste; NFI: índice de ajuste normado; CFI: índice de ajuste comparado; RMSEA: error de aproximación cuadrático. 


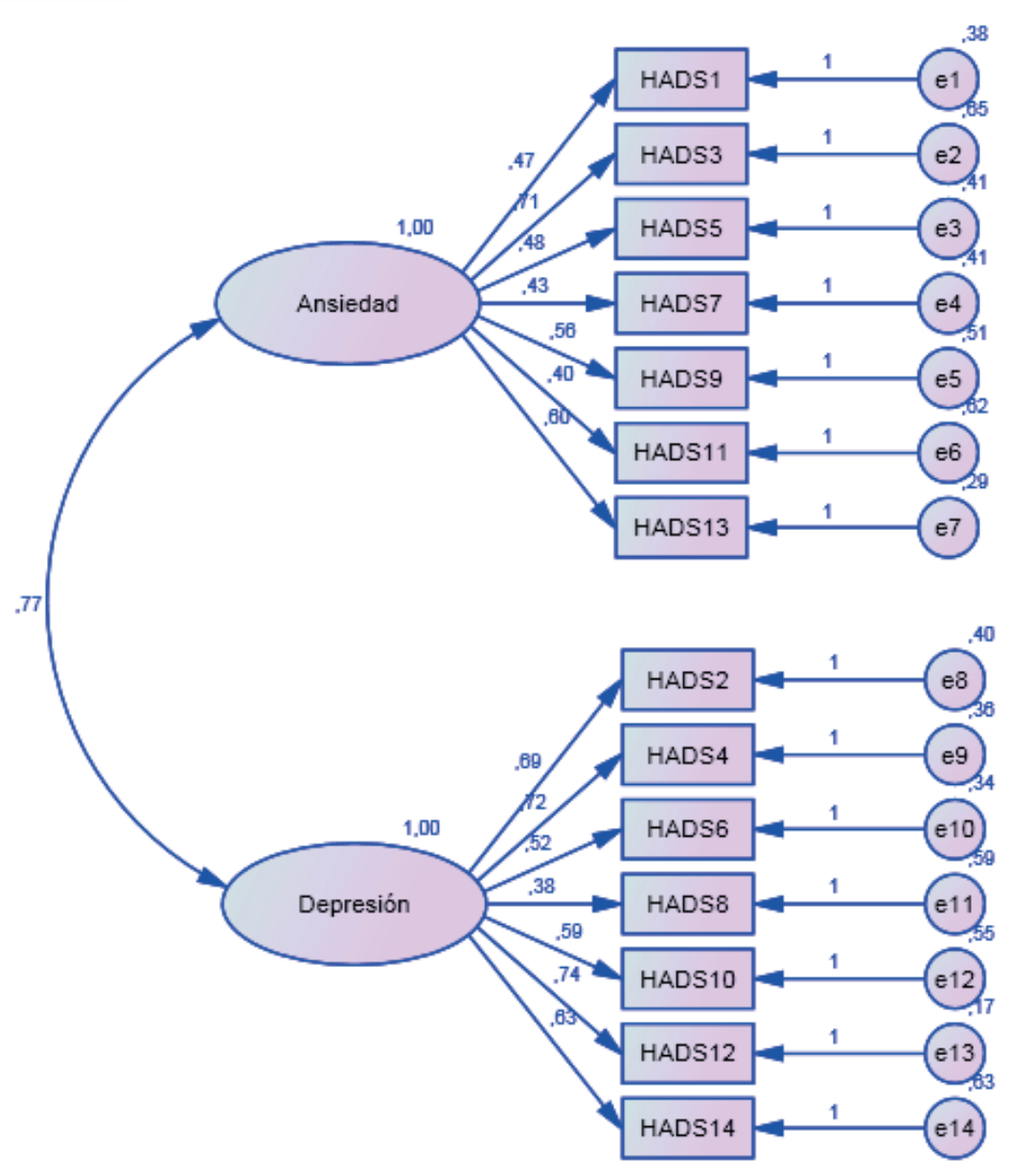

Figura 1. Modelo AFC bifactorial.

Tabla 5. Correlaciones item-total escalas del HAD.

\begin{tabular}{|c|c|c|}
\hline $\begin{array}{l}\text { HADA } \boldsymbol{\alpha}=.80 \\
\text { HADD } \boldsymbol{\alpha}=.85\end{array}$ & $\begin{array}{c}\text { Subescala } \\
\text { HADA }\end{array}$ & $\begin{array}{c}\text { Subescala } \\
\text { HADD }\end{array}$ \\
\hline 1. Me siento tenso/a o nervioso/a & .530 & \\
\hline 2. Sigo disfrutando con las mismas cosas de siempre & & 674 \\
\hline 3. Siento una especie de temor como si algo malo fuera a suceder & .501 & \\
\hline 4. Soy capaz de reírme y ver el lado gracioso de las cosas & & .669 \\
\hline 5. Tengo la cabeza llena de preocupaciones & .517 & \\
\hline 6. Me siento alegre & & .595 \\
\hline 7. Soy capaz de permanecer sentado/a tranquilo/a y relajadamente & .525 & \\
\hline 8. Me siento lento/a y torpe & & .449 \\
\hline 9. Experimento una desagradable sensación de "nervios y hormigueos en el estómago" & .562 & \\
\hline 10. He perdido el interés por mi aspecto personal & & .557 \\
\hline 11. Me siento inquieto/a como si no pudiera parar de moverme & .450 & \\
\hline 12. Tengo ilusión por las cosas & & .784 \\
\hline 13. de repente experimento sensaciones de gran angustia o temor & .632 & \\
\hline 14. Soy capaz de disfrutar con un buen libro o un buen programa de radio o de TV & & .594 \\
\hline
\end{tabular}


Tabla 6. Puntos de corte, sensibilidad y especificidad para cada criterio diagnóstico. Subescala Depresión.

\begin{tabular}{|c|c|c|c|c|c|c|c|c|c|}
\hline \multicolumn{10}{|c|}{ HADD } \\
\hline $\begin{array}{l}\text { CRITERIO } \\
\text { DIAGNÓSTICO }\end{array}$ & $\begin{array}{l}\text { SEGMENTO } \\
\text { DE CORTE }\end{array}$ & PUNTUACIÓN & $\begin{array}{c}\text { Punto } \\
\text { de } \\
\text { corte }\end{array}$ & $\begin{array}{c}\mathrm{S} \\
(\%)\end{array}$ & $\mathrm{E}(\%)$ & AUC & PPV (\%) & NPV (\%) & $\begin{array}{l}\text { Efectividad diag- } \\
\text { nóstica }(\mathrm{AC})\end{array}$ \\
\hline & & & 7 & 83 & 80 & .853 & 98 & 29 & 83 \\
\hline & Leve (10-18) & 10 & & & & & & & \\
\hline & & & 8 & 78 & 90 & .853 & 99 & 36 & 79 \\
\hline & Moderado (19-29) & 19 & 10 & 81 & 73 & .862 & 80 & 74 & 77 \\
\hline \multirow[t]{6}{*}{ BDI } & & & 11 & 78 & 71 & .837 & 66 & 80 & 74 \\
\hline & Dolor crónico & 21 & & & & & & & \\
\hline & & & 12 & 73 & 75 & .837 & 70 & 77 & 74 \\
\hline & & & 12 & 82 & 66 & .804 & 46 & 91 & 70 \\
\hline & Grave (30-63) & 30 & & & & & & & \\
\hline & & & 13 & 76 & 73 & .804 & 49 & 90 & 73 \\
\hline
\end{tabular}

AC: Accuracy Index (efectividad diagnóstica); AUC: Área Bajo la Curva; BDI: Inventario de Depresión de Beck (Beck Depression Inventory; 1978); E: Especificidad; HAD: Escala de Ansiedad y Depresión Hospitalaria de Zigmond y Snaith (Hospital Anxiety and Depression; 1983); HADA: Subescala de Ansiedad del HAD; HADD: Subescala de Depresión del HAD; NPV: Valor Predictivo Negativo; PPV: Valor Predictivo Positivo; S: Sensibilidad.

Tabla 7. Puntos de corte, sensibilidad y especificidad para cada criterio diagnóstico. Subescala Ansiedad.

\begin{tabular}{|c|c|c|c|c|c|c|c|c|c|}
\hline \multirow[b]{2}{*}{$\begin{array}{l}\text { CRITERIO } \\
\text { DIAGNÓSTICO }\end{array}$} & \multicolumn{9}{|c|}{ HADA } \\
\hline & $\begin{array}{l}\text { SEGMENTO } \\
\text { DE CORTE }\end{array}$ & PUNTUACIÓN & $\begin{array}{l}\text { Punto } \\
\text { de } \\
\text { corte }\end{array}$ & S (\%) & E (\%) & AUC & $\begin{array}{l}\text { PPV } \\
(\%)\end{array}$ & $\begin{array}{l}\text { NPV } \\
(\%)\end{array}$ & $\begin{array}{l}\text { Efectividad } \\
\text { diagnóstica } \\
\text { (AC) }\end{array}$ \\
\hline & & & 12 & 80 & 68 & .858 & 91 & 45 & 78 \\
\hline & Percentil 75 (M) & 31 & & & & & & & \\
\hline \multirow[t]{2}{*}{ STAI-E } & & & 13 & 74 & 84 & .858 & 96 & 43 & 76 \\
\hline & Percentil 90 (+1DT) & 44 & 16 & 72 & 68 & .755 & 27 & 94 & 69 \\
\hline
\end{tabular}

AC: Accuracy Index (efectividad diagnóstica); AUC: Area Bajo la Curva; E: Especificidad; HAD: Escala de Ansiedad y Depresión Hospitalaria de Zigmond y Snaith (Hospital Anxiety and Depression; 1983); HADA: Subescala de Ansiedad del HAD; HADD: Subescala de Depresión del HAD; NPV: Valor Predictivo Negativo; PPV: Valor Predictivo Positivo; S: Sensibilidad; STAI-E: Cuestionario de Ansiedad-Estado de Spielberger (State Trait Anxiety Inventary; 1970).

\section{Frecuencias y distribución de la muestra según puntos de corte. Prevalencia de ansiedady depresión}

Se comparó la distribución de frecuencias de nuestra muestra según los puntos de corte originales de Zigmond y Snaith y los obtenidos en este estudio (ver tablas 8-9).

En la subescala de depresión (HADD), siguiendo los puntos de corte propuestos por los autores originales, la muestra se distribuiría en $27.5 \%$ no caso, $21.4 \%$ posible caso y $51.1 \%$ caso. Según los puntos de corte de este estudio, un $21.4 \%$ sería no caso, un $20.6 \%$ depresión leve (o posible caso) y un $58 \%$ se considera como caso clasificado según gravedad del trastorno (ver tabla 8).
Para la subescala de ansiedad (HADA), la distribución de frecuencias para los puntos de corte originales es la siguiente: $6.9 \%$ no caso, $16.6 \%$ posible caso y $76.5 \%$ caso, frente a la distribución según los puntos de corte hallados en este estudio que es $29 \%$ no caso y $71 \%$ caso (en distintos niveles de gravedad de ansiedad) (ver tabla 9).

\section{Discusión}

En población española, algunos estudios ya han comprobado el rendimiento, las propiedades psicométricas y los puntos de corte del HAD en enfermos físicos (Costa et al., 2009; Herrero et al., 2003; López-Roig et al., 2000). En 
Tabla 8. Comparativa de la prevalencia depresión (puntos de corte originales-puntos de corte de muestra fibromiálgica).

\begin{tabular}{|c|c|c|c|c|c|c|c|}
\hline $\begin{array}{l}\text { Puntos de corte } \\
\text { Zigmond y Snaith } \\
\text { (1983) }\end{array}$ & Frecuencia & $\begin{array}{c}\% \\
\text { Prevalencia } \\
\text { muestra }\end{array}$ & $\begin{array}{c}\% \\
\text { Acumulado }\end{array}$ & $\begin{array}{l}\text { Puntos de corte en este estudio } \\
\text { (2014) }\end{array}$ & Frecuencia & $\begin{array}{c}\% \\
\text { Prevalencia } \\
\text { muestra }\end{array}$ & $\begin{array}{c}\% \\
\text { Acumulado }\end{array}$ \\
\hline \multirow{3}{*}{$>11:$ Caso } & \multirow{3}{*}{67} & \multirow{3}{*}{51,1} & \multirow{3}{*}{51,1} & $>12$ : Depresión grave & 61 & 40,5 & \multirow{3}{*}{58} \\
\hline & & & & $\begin{array}{l}>11: \text { Depresión con dolor } \\
\text { crónico }\end{array}$ & 14 & 10,6 & \\
\hline & & & & $>10$ : Depresión moderada & 9 & 6,9 & \\
\hline 8 a 10: Posible caso & 28 & 21,4 & 21,4 & 7 a 9: Depresión leve & 27 & 20,6 & 20,6 \\
\hline \multirow[t]{2}{*}{0 a $7:$ No caso } & 36 & 27,5 & 27,5 & 0 a 6: No caso & 28 & 21,4 & 21,4 \\
\hline & 131 & 100 & 100 & & 131 & 100 & 100 \\
\hline
\end{tabular}

Tabla 9. Comparativa de la prevalencia ansiedad (puntos de corte originales-puntos de corte de muestra fibromiálgica).

\begin{tabular}{|c|c|c|c|c|c|c|c|}
\hline $\begin{array}{l}\text { Puntos de corte Zigmond y Snaith } \\
\text { (1983) }\end{array}$ & Frecuencia & $\begin{array}{c}\% \\
\text { Prevalencia } \\
\text { muestra }\end{array}$ & $\begin{array}{c}\% \\
\text { Acumulado }\end{array}$ & $\begin{array}{l}\text { Puntos de } \\
\text { corte este es- } \\
\text { tudio (2014) }\end{array}$ & Frecuencia & $\begin{array}{c}\% \\
\text { Prevalencia } \\
\text { muestra }\end{array}$ & $\begin{array}{c}\% \\
\text { Acumulado }\end{array}$ \\
\hline \multirow{2}{*}{$>11:$ Caso } & \multirow{2}{*}{100} & \multirow{2}{*}{76,5} & \multirow{2}{*}{76,5} & $\begin{array}{l}>16: \text { Ansiedad } \\
\text { extrema }\end{array}$ & 49 & 37,4 & \multirow{2}{*}{71} \\
\hline & & & & $\begin{array}{l}>12 \text { : Ansiedad } \\
\text { significativa }\end{array}$ & 44 & 33,6 & \\
\hline \multirow[t]{2}{*}{8 a 10: Posible caso } & 22 & 16,6 & 16,6 & \multirow{4}{*}{0 a 11: No caso } & \multirow{3}{*}{38} & \multirow{3}{*}{29} & \multirow{3}{*}{29} \\
\hline & & & & & & & \\
\hline \multirow[t]{2}{*}{0 a 7 : No caso } & 9 & 6,9 & 6,9 & & & & \\
\hline & 131 & 100 & 100 & & 131 & 100 & 100 \\
\hline
\end{tabular}

FM, sólo en dos trabajos se evalúa el HAD, no llegando a analizar los puntos de corte óptimos para su uso en esta población (Luciano et al., 2013; Vallejo, Rivera, Esteve-Vives y Rodríguez-Muñoz, 2012). Sin embargo, el HAD es de gran interés para el caso de la FM, por la recomendación de la SER y por considerarse el "gold standard" para evaluar la depresión en estas pacientes (Boomershine, 2012; Luciano et al., 2013). Por todo ello, este estudio cobra especial relevancia, pues a los resultados de las propiedades psicométricas del HAD, se añade el análisis de su sensibilidad y especificidad, orientando así su aplicación y uso clínico en pacientes con FM.

En el caso de nuestras pacientes, la prevalencia de ansiedad y depresión es superior a un $70 \%$ y un $50 \%$ respectivamente, según nuestros puntos de corte $(\mathrm{HADD} \geq 10+/ 12+$; HADA $\geq 12+)$ (tablas 8 y 9). Este resultado coincide con lo referido en la literatura acerca de la presencia de estos trastornos en FM (Bennett, 2002; Epstein et al., 1999; González, Elorza y Failde, 2010; Revuelta, Segura y Paulino, 2010; entre otros. Estas pacientes suelen mostrar medias más elevadas en el HAD que las de otros enfermos físicos (Bambauer, Locke, Aupont, Mullan y McLaughlin, 2005; CooperEvans, Alderman, Kinht y Oddy, 2008; López-Roig et al., 2000; Montazeri, Vahdaninia, Ebrahimi y Jarvandi, 2003;
Rodríguez-Blazquez et al., 2009; Singer et al., 2009; entre otros) se acercan a las aportadas en dolor crónico (Keeky et al., 2008; Pallant y Bailey, 2005; Thomas et al., 2010; Wong et al., 2010; entre otros) y son similares a las de otras muestras con Síndrome de Fatiga Crónica (SFC) (Cella, Chalder y White, 2011; Crawley et al., 2013; Ledingham, Doherty y Doherty, 1993; Ray, Jefferies y Weir, 1995). La literatura aporta puntuaciones medias en el HAD para FM que oscilan en torno a 9-12 para ansiedad y 8-10 para depresión (rangos: 0-21) (Miró, Diener, Martínez, Sánchez y Valenza, 2014; Rodríguez-Muñoz, 2012; Scheafer et al., 2011; Nam, Tin, Bain, Thorne y Ginsburg, 2013; Vallejo et al., 2012). En concreto, nuestras medias de ansiedad y depresión con población española de FM se acercan a las de Vallejo et al. (2012) y Rodríguez-Muñoz (2012), siendo ambas más elevadas que las aportadas por Luciano et al. (2013).

Una posible explicación a estos resultados residiría, por un lado, en la propia enfermedad con dolor crónico y en las particularidades de la FM, y por otro lado, en el nivel asistencial del que proceden estas muestras de estudio. Así, las pacientes con FM, además de dolor generalizado, sufren un síndrome "multisintomático" con diagnóstico demorado, ineficacia de tratamientos, estrés crónico, incomprensión familiar y social, entre otros (Peñacoba, 2009; 
Revuelta, Segura y Paulino, 2010; Ubago, Ruiz, Bermejo, Olry y Plazaola, 2005). Todo ello deriva en un circuito asistencial y profesional marcado por la incertidumbre, el empeoramiento, y las pruebas o tratamientos que no logran solucionar su problema, lo que agravaría aún más su malestar psicológico (Peñacoba, 2009; Revuelta, Segura y Paulino, 2010). Podríamos decir que estas características de la FM, finalmente, "acompañan y propician" las respuestas de ansiedad y depresión más elevadas que se suelen mostrar, sobre todo en los niveles especializados donde las pacientes llegan como último recurso. Éste sería el caso de nuestro trabajo, el de Vallejo et al. (2012) o Miró et al. (2014), que realizados en centros asistenciales de fibromialgia o reumatología presentan respuestas de ansiedad y depresión más elevadas que los llevados a cabo en atención primaria (Luciano et al., 2013; Schaefer et al., 2011). El caso de las pacientes atendidas en los últimos niveles asistenciales o especializados, supone "a priori" una mayor problemática y gravedad asociada a su enfermedad y, como consecuencia, parecen esperables mayores niveles de ansiedad y depresión.

En este trabajo confirmamos la estructura factorial original del HAD (Zigmond y Snaith, 1983) en FM, al igual que Luciano et al. (2013), Vallejo et al. (2012) y otros trabajos realizados con diferentes enfermedades físicas en población española (Costa et al., 2009; Herrero et al., 2003; López-Roig et al., 2000). Además, la consistencia interna de las subescalas del HAD son superiores a .80 (HADA $\alpha=$ $.80, \operatorname{HADD} \alpha=.85$ ) en la línea de lo referido en la literatura (Bedford, Pauw y Grant, 1997; Constantini et al., 1999; Costa et al., 2009; Chivite et al., 2007; Herrero et al., 2003; Herrmann, 1997; López-Roig et al., 2000; Quintana et al., 2003; Rodríguez-Blázquez et al., 2009; Tejero et al., 1986; Vallejo et al., 2012; entre otros). En población española, la consistencia de la subescala HADA varía desde .74 (Monsalve et al., 2001) en pacientes ambulatorios de dolor crónico a .86 (Quintana et al., 2003) en una muestra mixta de enfermos crónicos y sanos. Para la subescala HADD se muestran valores entre .71 (Terol et al., 2007) en población sana y .87 (López-Roig et al., 2000) en población oncológica. En el caso del estudio con FM de Vallejo et al. (2012), su consistencia interna también es superior a .80 en ambas subescalas, y al igual que en nuestro caso, ligeramente inferior en ansiedad (de .83 , frente a .87 en depresión). Por su parte, las correlaciones ítem-test, similares para ambas subescalas, presentan valores semejantes a las de los trabajos de De las Cuevas et al. (1995), Terol et al. (2007) y Rodríguez-Blázquez et al. (2009). En nuestro estudio, los ítems 8 ("Me siento lento/a y torpe") y 11 ("Me siento inquieto/a como si no pudiera parar de moverme") fueron los de menor correlación ítem-test y carga factorial. A este respecto, una revisión de 15 estudios del HAD en muestra española (Terol, Cabrera y Martín-Aragón, 2015) mostró en cinco de ellos al ítem 8 como el de menor correlación, y en ocho trabajos fue el de menor carga factorial (Chivite et al., 2007; Herrero, et al., 2003; Monsalve et al., 2001; Quintana, et al., 2003; Rodríguez-Blázquez et al., 2009; Terol et al., 2007). Este análisis de ítems animaría a reevaluarlos más en detalle, con el objetivo de -si fuera posible- abreviar y optimizar aún más la aplicación del HAD en contextos de salud. Sin embargo, para algunos autores no deberíamos omitir ningún ítem, abogando por la complementariedad de sus subescalas (Luciano et al., 2013; Mitchell, Meader y Symonds, 2010; Norton, Cosco, Doyle, Done y Sacker, 2012; Sultan et al., 2010). Consideran que en la práctica clínica, la ansiedad podría ser un aspecto central de la depresión, por lo que el cómputo de ambas subescalas da sentido a un constructo más amplio; el "distrés psicológico" en el contexto del paciente crónico.

Los puntos de corte referidos como "óptimos" son los que muestran el mejor compromiso sensibilidad-especificidad y valores de predicción que avalan la capacidad diagnóstica de la prueba (Argimón y Jiménez, 2000; Burgueño, GarcíaBastos y González-Buitrago, 1995). Conforme a esto, para la subescala HADD sería el 10+ para depresión moderada y el 12+ para dolor crónico, según criterio del BDI. Éstos reflejan el mejor balance, mostrando valores superiores o cercanos al $70 \%$ en sensibilidad y especificidad y en todos los valores predictivos (AC, PPV, NPV). Respecto del resto de puntos de corte HADD-BDI, se obtienen buenos índices de sensibilidad, especificidad y efectividad diagnóstica (AC), pero varían los valores predictivos positivos y negativos. En la subescala del HADA sería el punto de corte 12+, del percentil 75 (ansiedad clínicamente significativa), el que obtiene mejor índice pronóstico, con sensibilidad de $80 \%$, especificidad del $68 \%$ y eficacia diagnóstica del $78 \%$ (PPV $=91 \%$; NPV=45\%).

Un aspecto que cabe abordar aquí es la variabilidad de los puntos de corte del HAD, a través de distintos estudios. Esto ha fomentado en ocasiones el debate y la controversia acerca de la utilidad y eficacia del HAD, de sus subescalas y de su uso como escala global de distrés psicológico (Brennan et al., 2010; Coyne y Van Sonderen, 2012; Mitchell, Meader y Symonds, 2010; Norton, Cosco, Doyle, Done y Sacker, 2012). Probablemente, esta variabilidad de puntos de corte del HAD tiene que ver fundamentalmente con dos aspectos: 1) los instrumentos criterio, representatividad de los componentes de depresión y/o ansiedad evaluados (cognitivos y/o afectivos, o incluso comportamentales) y teorías que 
respaldan estos constructos, y 2) la heterogeneidad de las muestras de estudio. Así, por ejemplo, los criterios BDI y CES-D utilizados en diferentes estudios, aún compartiendo algunos síntomas, medirían distintos aspectos de la depresión (Skorikov y Van der Voort, 2003). Entre sus diferencias, mientras el BDI tiene mayor peso cognitivo, el CES-D se inclina hacia el componente afectivo de la depresión (Gempp, Avendaño y Muñoz, 2004; Sultan et al., 2010). Por su parte, en el BDI o el STAI, una mayor presencia de ítems somáticos (fatiga, alteraciones del sueño, entre otros) podría "sobreestimar" la sintomatología ansiosa-depresiva, y en consecuencia, elevar los puntos de corte del HAD (Comeche, Ortega, Rodríguez-Muñoz, Díaz y Vallejo, 2012). Sin embargo, en nuestra opinión, en FM este "solapamiento" diagnóstico aporta información de la propia realidad de las pacientes. Y quizás no podamos, pero tampoco debamos, desvincular la sintomatología físico-psicológica, ya que con ella como un "todo" hemos de trabajar y/o intervenir clínicamente en FM.

Respecto de la heterogeneidad muestral con el HAD, comparando nuestros puntos de corte con los obtenidos por Costa et al. (2009) y López-Roig et al. (2000) en oncología y Herrero et al. (2003), éstos oscilan entre 8+-9+ para el HADA y entre $3+-5+$ para el HADD, frente a $12+$ para nuestra subescala de ansiedad, y de 10+ ó 12+ para depresión. Esta diferencia se explicaría desde la prevalencia de sintomatología ansioso-depresiva en ellas, pues en otros enfermos físicos e incluso oncológicos no es tan elevada (Costa et al., 2009; Fernández et al., 2006; López-Roig et al., 2000; Singer et al., 2009). Sin embargo, en el caso de FM, como ya hemos comentado, sí lo es y se acerca a la del Síndrome de Fatiga Crónica (SFC) (Ledingham, Doherty y Doherty, 1993; Ray, Jefferies y Weir, 1995; entre otros), patología con la que comparte mecanismos fisiopatológicos, síntomas y consecuencias emocionales, entre otros. De hecho, pacientes de SFC también obtienen puntos de corte para HADD en torno a 10+ (S:92\%, E:71\%) (Morris y Wearden, 1998; y Henderson y Tannock, 2005). Por otro lado, y siguiendo con la heterogeneidad muestral, sociodemográfica en este caso, en nuestro trabajo el total de participantes con FM son mujeres. Otros trabajos incluyen un mínimo porcentaje de hombres que, sin embargo, introducen ciertas variaciones en las medias y puntos de corte hallados, lo que debe tenerse en cuenta a la hora de extrapolar los resultados de las investigaciones con FM (Cabrera et al., 2012).

A modo de resumen, se destaca que nuestras elevadas medias de FM en ansiedad y depresión y puntos de corte propuestos son similares a los de otros estudios específicos con FM, o patologías afines como SFC. Los "casos" encontrados comparten el mismo rango originalmente propuesto por Zigmond y Snaith, lo que hace que, de entre los estudios realizados con una muestra española de enfermos físicos, el nuestro sea el más cercano al original (Costa et al., 2009; López-Roig et al., 2000; Herrero et al., 2003).

De los análisis de sensibilidad y especificidad con curvas ROC, se muestra un HAD útil para el diagnóstico de ansiedad y depresión en FM. En ambas subescalas, los datos de las AUC son idóneos, por encima del $75 \%$, lo que significaría que un paciente seleccionado aleatoriamente como caso por cualquiera de los criterios externos, lo sería también en el HAD más de un $75 \%$ de las veces.

En relación a los instrumentos criterio (BDI, STAI-E), sería deseable complementarlos con criterios DSM y mediante entrevista clínica pero, ciertamente, parece poco viable pues en contextos clínicos es prioritario aplicar procedimientos con el menor coste posible, rápidos y eficaces en el diagnóstico, sin sobrecargar la dinámica del servicio de salud o unidad, ni someter al paciente a pruebas, visitas $\mathrm{y}$ tratamientos que pueden percibirse como infructuosos, más aun en patologías crónicas como la FM.

Respecto a la validez externa de los resultados, un aspecto a considerar para futuras investigaciones es contrastar resultados en el HAD con submuestras representativas que permitan comparar entre variables sociodemográficas y clínicas determinadas (género, grupos de edad, asistencia especializada vs. primaria, entre otras). Es necesario el cálculo de puntos de corte en atención primaria con pacientes de FM que no han llegado a unidades del dolor o fibromialgia, de diagnóstico reciente o de distinta tipología. Esto permitiría evaluar en qué medida la cronicidad o gravedad, alto estrés, incertidumbre y síntomas de ansiedad y depresión por un tratamiento prolongado o un diagnóstico demorado en el tiempo "sesga" la evaluación de la población general de FM (Bennet, 2002; Peñacoba, 2009).

Para terminar, nuestros resultados siguen la línea de los encontrados con el HAD en otros trabajos, lo que consolida su consistencia y validez para evaluar y diagnosticar ansiedad y depresión también en pacientes con FM. El alto porcentaje de coincidencia entre casos identificados por el HAD en relación con otros instrumentos con mayor tradición como BDI o STAI y las buenas propiedades psicométricas mostradas, nos hace valorar su idoneidad para la población estudiada. La coherencia al describir la prevalencia de ansiedad y depresión (ver tablas 8 y 9), comparando los puntos de corte del estudio y los originales (Zigmond y Snaith, 1983), apuntan también en la dirección del HAD como un buen instrumento para FM. Pero además, la brevedad y aceptación del instrumento, el mostrarse fiable 
y válido manteniendo su estructura factorial original, y la recomendación de la SER lo hacen imprescindible en el contexto de la FM. En este trabajo se sugieren puntos de corte para cada subescala (ver tablas 6 y 7), pero conviene recordar que el HAD ha demostrado su utilidad con el uso de su puntuación total en diferentes muestras (Brennan et al., 2010; Cosco, Doyle, Ward y McGee, 2011) y recientemente también en FM (Luciano et al., 2013). La posibilidad de valorar globalmente el distrés psicológico, junto al recurso de sus subescalas específicas, en nuestra opinión añade versatilidad permitiendo ajustar los intereses y objetivos de la evaluación clínica (Terol et al., 2007).

\section{Referencias}

Álvarez, R. (2007). Estadística aplicada en ciencias de la salud. Madrid: Díaz de Santos.

Argimon, J. M. y Jiménez, J. (2000). Métodos de investigación clínica y epidemiológica. Barcelona: Harcourt.

Bambauer, K. Z., Locke, S. E., Aupont, O., Mullan, M. G. y McLaughlin, T. J. (2005). Using the Hospital Anxiety and Depression Scale to screen for depression in cardiac patients. General Hospital Psychiatry, 27, 275-284.

Beck, A.T. (1978). Depression inventory. Filadelfia: Center for Cognitive Therapy.

Beck, A.T., Steer, R.A. y Harbin, M.G. (1988). Psychometric properties of the Beck Depression Inventory: Twenty-five years of evaluation. Clinical Psychology Review, 8, 77-100.

Bedford, A., Pauw, K. D. y Grant, E. (1997). The structure of the Hospital Anxiety and Depression Scale (HAD): an appraisal with normal, psychiatric and medical patient subjects. Personal Individual Differences, $23,473-8$.

Bennet, R. M. (2002). Rational Management of fibromyalgia. Rheumatic Disease Clinics of North America, 28, 13-15.

Bjelland, I., Dahl, A. A., Haug, T. T. y Neckelmann, D. (2002). The validity of the Hospital Anxiety and Depression Scale. An updated literature review. Journal of Psychosomatic Research, 52, 69-77.

Boomershine, C. S. (2012). A comprehensive evaluation of standardized assessment tools in the diagnosis of fibromyalgia and in the assessment of fibromyalgia severity. Pain Research and Treatment. 1-11. doi:10.1155/2012/653714.

Brennan, C., Worrall-Davies, A., McMillan, D., Gilbody, S. y House, A. (2010). The Hospital Anxiety and Depression Scale: A diagnostic metaanalysis of case-finding ability. Journal of Psychosomatic Research, 69, 371-378.

Burgueño, M. J., García-Bastos, J. L. y González-Buitrago, J.M. (1995). Las curvas ROC en la evaluación de las pruebas diagnósticas. Medicina Clínica, 104, 661-70.

Byrne, B. M (2010). Structural equation modeling with AMOS: Basic concepts, application and programming 2nd Edition. New York: Taylor y Francis Group.

Cella, M., Chalder, T. y White, P. D. (2011). Does the heterogeneity of chronic fatigue syndrome moderate the response to cognitive behaviour therapy? An exploratory study. Psychotherapy and Psychosomatics, 80, 353-358. doi:10.1159/000327582.

Chivite, S., Martínez, M., Pérez, E. y Peralta, V. (2007). HADS: Estudio de criterios de validez interna y externa en una muestra española de pacientes hospitalizados. Cuadernos de Medicina Psicosomática y Psiquiatría de Enlace, 84, 9-17.
Comeche, M. I., Martín, A., Rodríguez-Muñoz, M. F., Ortega, J., Díaz, M. I. y Vallejo, M. A. (2010). Tratamiento cognitivo-conductual, protocolizado y en grupo, de la fibromialgia. Clínica y Salud, 21, 107-121.

Comeche, M. I., Ortega, J., Rodríguez-Muñoz, M. F., Díaz, M. I. y Vallejo, M. A. (2012). Estructura y adecuación del inventario de depresión de Beck en pacientes con fibromialgia. Psichotema, 24, 668-673.

Cooper-Evans, S., Alderman, N., Kinht, C. y Oddy, M. (2008). Self-esteem as predictor of psychological distress after severe acquired injury: an exploratory study. Neuropsychology Rehabilitation, 18, 607-626.

Cosco, T., Doyle, F., Ward, M. y McGee, H. (2011). Latent structure of the hospital anxiety and depression scale: a 10 year systematic review. Psychology Articles. doi:10.1016/j.jpsychores.2011.06.008.

Costa, G., Pérez, X., Salamero, M. y Gil, F. L. (2009). Discriminación del malestar emocional en pacientes oncológicos utilizando la Escala de Ansiedad y Depresión Hospitalaria (HADS). Ansiedad y Estrés, 15, 217-229.

Constantini, M., Musso, M., Viterbori, P., Bonci, F., Del Mastro, L., Garrone, O., Venturini, M. y Morasso, G. (1999). Detecting psychological distress in cancer patients: validity of the Italian version of the Hospital Anxiety and Depression Scale. Supportive Care Cancer, 7, 121-127.

Coyne, J. C. y Van Sonderen, E. (2012). No further research needed: Abandoning the Hospital and Anxiety Depression Scale (HADS). Journal of Psychosomatic Research, 72, 173-174. doi:10.1016/j. jpsychores.2011.12.003.

Crawley, E., Collin, S. M., White, P. D., Rimes, K., Sterne, J. A. C. y May, M. T. (2013). Treatment outcome in adults with chronic fatigue syndrome: a prospective study in England based on the CFS/ME National Outcomes Database. QJM, hct061. doi:10.1093/qjmed/hct061.

De las Cuevas, C., García-Estrada, A. y González de Rivera, J. L. (1995). Hospital Anxiety and Depresión Scale y Psicopatología Afectiva. Anales de Psiquiatría, 2, 126-130.

Echeburúa, E. (2004). Superar un trauma. Madrid: Pirámide.

Epstein, S. A., Kay, G., Clauw, D., Heaton, R., Klein, D., ...Krupp, L. (1999). Psychiatric disorders in patients with fibromyalgia. Psychosomatics, 40, 57-63. doi: 10.1016/S0033-3182(99)71272-7.

Esteve-Vives, J., Batlle Gualda, E. y Reig, A. Grupo para la adaptación del HAQ a la población española (1993). Spanish Version of the Health Asessment Questionnaire: reliability, validity and transcultural equivalency. Journal of Rheumatology, 20, 2116-2122.

Fernández, C., Padierna, C., Amigo, I., Pérez, M., Gracia, J. M., Fernández, R. y Peláez, I. (2006). Calidad de vida informada por pacientes oncológicos paliativos: Relaciones con el informe médico y estado emocional. Index de Enfermería, 15, 30-34. doi:10.4321/S113212962006000100007.

García-Cueto, E., Gallo-Álvaro, P. y Miranda, R. (1998). Bondad de ajuste en el análisis factorial confirmatorio. Psicothema, 10, 717-724.

Geisser, M. E., Roth, R. S. y Robinson, M.E. (1997). Assessing depression among persons with chronic pain using the Center for Epidemiological Studies-Depression Scale and the Beck Depression Inventory: a comparative analysis. Clinical Journal of Pain, 13, 163-170.

Gempp, R., Avendaño, C. y Muñoz, C. (2004). Normas y punto de corte para la Escala de Depresión del Centro para Estudios Epidemiológicos (CES-D) en población juvenil chilena. Terapia Psicológica, 22, 145 156.

González, E., Elorza, J. y Failde, I. (2010). Comorbilidad psiquiátrica y fibromialgia. Su efecto sobre la calidad de vida de los pacientes. Actas de Psiquiatría Española, 38, 295-300.

Hamilton, M. y Shapiro, C. M. (1990). Depression. En Peck D.F. y Shapiro C.M. (coords.). Measuring human problems: a practical guide. New York: Wiley.

Herrero, M. J., Blanch, J., Peri, J. M., De Pablo, L., Pintor, A. y Bulbena, A. (2003). A validation study of the Hospital anxiety and depression scale (HADS) in a Spanish population. General Hospital Psychiatry, $25,277-283$.

Herrmann, C. (1997). International Experiences with the Hospital Anxiety and Depression Scale-A review of Validation data and Clinical results. Journal of Psychosomatic Research, 1 (42), 17- 41. 
Henderson, M. y Tannock, C. (2005). Use of depression rating scales in chronic fatigue syndrome. Journal Psychosomatic Research, 59, 181-184.

Ibáñez, E. y Caro, I. (1992). La escala Hospitalaria de ansiedad y depresión. Su utilidad práctica en Psicología de la Salud. Boletín de Psicología, 36, 43-69.

Katon, W. (1996). The impact of major depression on chronic medical illness. General Hospital Psychiatry, 18, 215-219.

Keeley, P., Creed, F., Tomenson, B., Todd, C., Borglin, G. y Dickens, C. (2008). Psychosocial predictors of health-related quality of life and health service utilisation in people with chronic low back pain. Pain, 135, 142-150. doi:10.1016/j.pain.2007.05.015.

Ledingham, J, Doherty, S. y Doherty, M. (1993). Primary fibromyalgia syndrome. An outcome study. British Journal of Rheumatology, 32, 139-142.

López-Roig, S., Terol, M. C., Pastor, M. A., Massutí, B., Rodríguez-Marín, J., Neipp, M.C., Leyda, J.I., Martín-Aragón, M., Sánchez, S. y Sitges, E. (2000). Ansiedad y Depresión. Validación de la escala HAD en pacientes oncológicos. Revista de Psicología de la Salud, 2, 127-157.

Luciano, J. V., Barrada, J. R., Aguado, J., Osma, J. y García-Campayo, J. (2013). Bifactor Analysis and Construct Validity of the HADS: A CrossSectional and Longitudinal Study in Fibromyalgia Patients. Psychological Assessment. Advance online publication. doi:10.1037/a0035284.

Mykletun, A., Stordal, E. y Dahl, A. A. (2001). Hospital Anxiety and Depression (HAD) scale: factor structure, item analyses and internal consistency in a large population. British Journal of Psychiatry, 179, 540-544.

Miró, J. (2003). Dolor Crónico: procedimientos de evaluación e intervención psicológica. Bilbao: Desclée de brouwer.

Miró, E., Diener, F. N., Martínez, M. P., Sánchez, A. I. y Valenza, M. C. (2014). La fibromialgia en hombres y mujeres: comparación de los principales síntomas clínicos. Psicothema, 24, 10-15.

Mitchell, A.J., Meader, N. y Symonds, P. (2010). Diagnostic validity of the Hospital Anxiety and Depression Scale (HADS) in cancer and palliative settings: A meta-analysis. Journal of Affective Disorders, 126, 335-348.

Moix, J. (2005). Análisis de los factores moduladores del dolor crónico benigno. Anuario de Psicología, 36, 37-60.

Monsalve V., Cerdá-Olmedo G., Mínguez, A. y De Andrés, J. A. (2000). Anxiety and depression in patients with chronic pain receiving home care opposite to outpatient care. Revista de la Sociedad Española del Dolor, 7, 6-11.

Monsalve, V., Soriano, J. e Ibáñez, E. (2001). Fiabilidad y estructura factorial de la escala de ansiedad y depresión hospitalaria (HAD) en una muestra de pacientes con dolor crónico. Boletín de Psicología, 72, 7-21.

Montazeri, A., Vahdaninia, M., Ebrahimi, M. y Jarvandi, S. (2003). The Hospital Anxiety and Depression Scale (HADS): translation and validation study of the Iranian version. Health and Quality of Life Outcomes, 1, 1-5. doi:10.1186/1477-7525-1-14

Morriss, R. K. y Wearden, A. J. (1998). Screening instruments for psychiatric morbidity in chronic fatigue syndrome. Journal of the Royal Society of Medicine, 91, 365-368.

Morse, R., Kendell, K. y Barton, S. (2005). Screening for depression in people with cancer: the accuracy of the hospital anxiety and depression scale. Clinical Effectiveness in Nursing, 9, 188-196

Nam, S., Tin, D., Bain, L., Thorne, J. C. y Ginsburg, L. (2013). Clinical utility of the Hospital Anxiety and Depression Scale (HADS) for an Outpatient Fibromyalgia Education Program. Clinical Rheumatology. doi:10.1007/s10067-013-2377-1.

Noguera, A., Centeno, C., Carvajal, A. Portela, M.A., Urdiroz, J. y Martínez, M. (2009). Spanish "Fine Tuning" of Language To Describe Depression and Anxiety. Journal of Palliative Medicine, 12, 707-712.

Norton, S., Cosco, T., Doyle, F., Done, J. y Sacker, A. (2012). The Hospital Anxiety and Depression Scale: A meta confirmatory factor analysis. Journal of Psychosomatic Research, 74, 74-81.

Pallant, J. F. y Bailey, C. M. (2005). Assessment of the structure of the Hospital Anxiety and Depression Scale in musculoskeletal patients. Health and Quality of Life Outcomes, 3, 82. doi:10.1186/1477-7525-3-82.
Peñacoba, C. (2009). Intervención psicológica en personas con Fibromialgia. Infocop, Revista de Psicología, 43,14- 20.

Pérez-Pareja, J., Borrás, R., Palmer, A., Sesé, A., Molina, F. y Gonzalvo, J. (2004). Fibromialgia y emociones negativas. Psicothema, 16, 415-420.

Quintana, J. M., Padierna, A., Esteban, C., Arostegui, I., Bilbao, A. y Ruiz, I. (2003). Evaluation of the Psychometric characteristics of the Spanish version of the Hospital Anxiety and Depression Scale. Acta Psychiatrica Scandinavica, 10, 216-221.

Ray, C., Jefferies, S. y Weir, W.R.C. (1995). Life-events and the course of the chronic fatigue syndrome. British Journal of Medical Psychology, $68,323-331$.

Revuelta, E., Segura, E. y Paulino, J. (2010). Depresión, ansiedad y fibromialgia. Revista de la Sociedad Española del Dolor, 17, 326-32.

Rivera, J., Alegre, C., Ballina, F., Carbonell, J., Carmona, L., ... Castel, B. (2006). Documento de consenso de la Sociedad Española de Reumatología sobre la fibromialgia. Reumatología Clínica, 2, 55-66.

Rivera, J., Rejas, J., Esteve-Vives, J., Vallejo, M. A. y Grupo ICAF (2009, mayo). Costes económicos asociados con el diagnóstico de Fibromialgia. Póster presentado en el XXXV Congreso de la SER (Sociedad Española de Reumatología).

Rodríguez-Blázquez, C., Frades-Payo, B., Forjaz, M. J., de Pedro-Cuesta, J., Martínez-Martín, P. y Longitudinal Parkinsons Dis Patien Group. (2009). Psychometric Attributes of the Hospital Anxiety and Depression Scale in Parkinson's Disease. Movement Disorders, 24,519-525.

Rodríguez-Muñoz, M. F., Comeche, J., Ortega, M., Díaz, y Vallejo, M. A. (2012, septiembre). Intervención grupal en enfermos de fibromialgia. IX Congreso Internacional para el Estudio de la Ansiedad y el Estrés. Valencia, España.

Ruiz, R., Muñoz, M., Pérula de Torres, L. y Aguayo, M. A. (1997). Biopsychosocial features of patients with widespread chronic musculoskeletal pain in family medicine clinics. Family Practice, 14, 242-248.

Schaefer, C., Chandran, A., Hufstader, M., Baik, R., McNett, M., Goldenberg, D. y Zlateva, G. (2011). The comparative burden of mild, moderate and severe fibromyalgia: results from a cross-sectional survey in the United States. Health and Quality of Life Outcomes, 9, 71. doi:10.1186/1477-7525-9-71.

Sicras, A., Blanca, M., Navarro, R. y Rejas, J. (2009a). Perfil de uso de recursos y costes en pacientes que demandan atención por fibromialgia o trastorno de ansiedad generalizada en el ámbito de la atención primaria de salud. Atención Primaria, 41, 77-84

Sicras, A., Rejas, J., Navarro, R., Blanca, M., Morcillo, A., Larios, R., Velasco, S. y Villarroya, C. (2009b). Treating patients with fibromylagia in primary care settings under routine medical practice: a claim database cost and burden of illness study. Arthritis Research y Therapy, 11, 54-69.

Singer, S., Kuhnt, S., Gotze, H., Hauss, J., Hinz, A., Liebmann, A. y Schwarz, R. (2009). Hospital anxiety and depression scale cutoff scores for cancer patients in acute care. British Journal Cancer, 100, 908-912.

Skorikov, V. B. y Van der Voort, D. J. (2003). Relationships between the underlying constructs of depression of the Beck Depression Inventory and the Center for Epidemiological Studies Depression Scale. Educational and Psychological Measurement, 63, 319-335.

Soler, J., Pérez, V., Puigdemont, D., Pérez, J. Figueres, M. y Álvarez, E. (1997). Estudio de validación del Center for Epidemiologic Studies Depression Scale (CES-D) en una población española de pacientes con trastornos afectivos. Actas Luso Españolas de Neurología y Psiquiatría $y$ Ciencias afines, 25(4), 243-249.

Spielberger, C. D., Gorsuch, R. L. y Lushene, R. (1982). Manual del Cuestionario de Ansiedad Estado/Rasgo (STAI) (3a edic.). Madrid: TEA. Spinhoven, P. H., Ormel, J., Sloekers, P. P., Kemper, G. I., Speckens, A. E. y Van Hemert, A. M. (1997). A validation study of the hospital Anxiety and Depression Scale in different groups of Dutch subjects. Psychol med, 27, 363-370.

Sultan, S., Luminet, O. y Hartemann, A. (2010). Cognitive and anxiety symptoms in screening for clinical depression in diabetes: A systematic examination of diagnostic performances of the HADS and BDI-SF. Journal of Affective Disorders, 123, 332-336. 
Tejero, A., Guimera, E., Farré, J. M. y Peri, J. M. (1986). Uso clínico del HADS (Hospital Anxiety and Depression Scale) en población psiquiátrica: un estudio de sensibilidad, fiabilidad y validez. Revista del Departamento de Psiquiatría, Facultad de Medicina, U. de Barcelona, 12, 233-238.

Terol, M. C., Cabrera, V. y Martín-Aragón, M. (2015). Revisión de estudios de la Escala de Ansiedad y Depresión Hospitalaria (HAD) en muestras españolas. Anales de Psicología, 31 (2), 494-503. http://dx.doi. org/10.6018/analesps.31.2.172701.

Terol, M.C., López-Roig, S., Rodríguez-Marín, J., Martín-Aragón, M., Pastor, M. A. y Reig, M. T. (2007). Propiedades psicométricas de la Escala Hospitalaria de Ansiedad y Estrés (HAD) en población española. Ansiedad y Estrés, 13, 163-176.

Thomas, E. N., Pers, Y. M., Mercier, G., Cambiere, J. P., Frasson, N., Ster, F. y Blotman, F. (2010). The importance of fear, beliefs, catastrophizing and kinesiophobia in chronic low back pain rehabilitation. Annals of Physical and Rehabilitation Medicine, 53, 3-14. doi:10.1016/j. rehab.2009.11.002.

Tyrer, P. y Methuen, C. (2007). Rating Scales in Psychiatry. London: RCPsych Publications.

Ubago, M. C., Ruiz, I., Bermejo, M. J., Olry, A. y Plazaola, J. (2005). Características clínicas y psicosociales de personas con fibromialgia. Repercusión del diagnóstico sobre sus actividades. Revista Española de Salud Pública, 79, 683-695.

Valverde, M. (2007). Prevalencia de la fibromialgia en la población española. Estudio EPISER. Revista Española de Reumatología, 27, 57.

Wong, W. S., Kwok, H. Y., Luk, K. D. K., Chow, Y. F., Mak, K. H., Tam, B. K. H., ... Fielding, R. (2010). Fear of movement/(re)injury in Chinese patients with chronic pain: Factorial validity of the Chinese version of the Tampa Scale for Kinesiophobia. Journal of Rehabilitation Medicine, 42, 620-629. doi:10.2340/16501977-0575.

Vallejo, M. A., Rivera, J., Esteve-Vives, J. y Rodríguez-Muñoz, M. F. (2012). Uso del cuestionario Hospital Anxiety and Depression Scale (HADS) para evaluar la ansiedad y la depresión en pacientes con fibromialgia. Revista de Psiquiatría y Salud Mental, 5, 107-114.

Vázquez, C. y Sanz, J. (1997). Fiabilidad y validez de la versión española del Inventario para la Depresión de Beck de 1978. Clínica y Salud, 8, 403-422.

Vázquez, F. L., Blanco, V. y López, M. (2007). An adaptation of the Center for Epidemiologic Studies Depression Scale for use in non-psychiatric Spanish populations. Psychiatry Research, 15, 247-52.

Wolfe, F. et al. (1990). The American College of Rheumatology 1990. Criteria for the classification of Fibromyalgia. Arthritis and Rheumatism, 33, 161-172.

Wolfe, F., Anderson, J., Harkness, D., Bennet, R.M., Caro, X.J., Goldenberg, D.L., Russell, I.J. e Yunus, M.B. (1997). A prospective, longitudinal, multicenter study of services utilization and cost in fibromyalgia. Arthritis and Rheumatism, 40, 1560-1570.

Zigmond, A. y Snaith, R. (1983). The Hospital Anxiety and Depression Scale. Acta Psychiatrica Scandinavica, 67, 361-370. 
